\title{
INDEX
}

Acacia, 57 sqq.

recessives in, 60 sqq.

linkage in, 63

acclimatisation, temperature, 282 development, 282

adaptation, 157

adaptive value, 277

affinity, 127

agglutination, 73

Agropyron, 129 sqq.

alleles, association of, I I

distribution of, $\mathrm{I} I$

pseudo-, 234, 280

multiple, 276

self-sterility, 276

semi-dominant, 279

semi-lethal, 253

Allison, A. C., 440

Allium, 37 I

ameiosis, 135

amphidiploid, 319

Andrewartha; H. G., 120

animal numbers, 120

antheridium, 248 sqq.

Anthoxanthum, 201, 363

antigen, 285, 427

Enothera, 255

apogamy, 253

apomixis, 131 sqq.

apospory, 157,253

archegonium, 247,248

array statistics, 12

ascospore, 280

Aspergillus, 124, 234, 254, 279, 280

Asplenium, 253

assimilation, genetic, $28 \mathrm{r}$

assortative mating, 323

Astroloma, 441

asymmetry, bilateral, 419, 283

asynapsis, I 51 sqq., 409

Auerbach, C., 28 I

autogamy, 257

autosomal lethals, mice, 123

Avena, Iog sqq.

backcross, I sqq., 225 sqq.

triplex, 208, 2 1 2

back-mutation, 279

bacteria, 435

balance, internal, 244

relational, 244

balanced lethal systems, 265

Bartlett, test, 4 I 5

Bauer, 435

Bateman, A. J., 257

Bateman, K. G., 281

B-chromosome, 195, 345, 355

Bennet, H. J., 278

Bennett, J. H., 51-55, 263-270

Binet, F. E., 5 1-55
Birch, L. C., $120-121$

Bird, G. W. G., 425-429

birth control, 278

Biston betularia, 287 sqq., 439

Blackwood, M., 353

blood groups, 165

$\mathrm{ABO}, 69$ sqq., 126

Duffy, 285

duodenal ulcer and, 126

frequencies, Ireland, $69 s q q$.

gene frequencies, 285

poultry, 243

race and, 16r sqq., 285, 425 sqq.

Rhesus, 69 sqq., 285, 425

sex and, 777

blood transfusion, 72, 285

Bombyx, 398

body size, Drosophila, 125

bracken, incompatability in, $247 s q q$.

Breese, E. L., 323-343

Brown, A. G., 237-245

Calef, E., 279

Caltha, 201

camouflage, 439

Campanula, 99, 370

Carter, T. C., 284

Catcheside, D. G., 205-21 8, 345-351

Centaurea, 201, 202

centromere, 99, 119, 127, 208, 217, 229, $284,345,382$

abnormal, 200

interference, $23 \mathrm{I}$

terminal, $197 \mathrm{sqq}$.

Cheiranthus, 257

chromatography, 280

chromocentres, 367,373

chiasma, $151,284,380$ sqq.

frequency, 156, 232, 234, 409, sqq.

chromatid, 229, 345, 350, 386

aberrations, 123

segregation, 105 sqq.

chromomere, 434

chromonema, I 24

chromosome, aberrations, 129 sqq., 385, 431

accessory, 195

acentric, $112,384 s q q$.

behaviour, 99, 409 sqq.

breakage, 199, 367 sqq.

complement, 119,268

deficiency, 156

dicentric, $156,3^{84} s q q$.

differentiation, $85 \mathrm{sqq}$.

distribution, 197, 367 sqq.

disjunction, 99, 354 sqq.

doubling, 85, 94, 109, 231, 349

fragment, 112, 195 sqq., 371 sqq.

genetical control, 99

genetics of $\mathrm{B}, 345$ sqq., 353 sqq.

homologous, 263,370 
chromosome, lagging, 148 sqq.

loss of, 109

maize, 345 sqq., 353 sqq.

morphology, 88, I $19,367,370$

neocentric, 153

number evolution, 284

pairing, 156

relational coiling, $15 \mathrm{I}$

ribbon, 44

ring, 133

spiralisation, I I 2, I $5^{1}, 374$ sqq.

sticky, II I

supernumerary, 195

synthesis, I 13 , I 14

telocentric, $197,3^{86}$

Circotettix, 200

Clarke, C. A., 126

Clayton, G. A., 282

clone, bracken, 248 sqq.

clover, $44^{\circ}$

Colopa, 126

colchicine, 109

combining ability, 3 I sqq.

complementary genes, $1,27 s q q$.

components of variation, $18 \mathrm{sqq}$.

linkage, 23 sqq.

cotton, selection in, 303 sqq.

correlation, between gametes, 277

phenotypic, 125,329 sqq.

genetic, 323 sqq.

co-variance between relatives, 31,35

Cragg, J. B., 431

Crosby, J. L., 125

crossing-over, 227 sqq., 265, 367, 378

inversion, 133

Crucifere, $26 \mathrm{I}$

crypsis, 293 sqq.

cytoplasm, 280

cytoplasmic gradient, I 4

Dahlia, 229

Darlington, C. D., $44^{1}$

Darwin, 433

Datura stramonium, 229

Davidson, D., 439

Dawson, C. J., 69-84

Dawson, G. W. P., 69-84, 285

deoxyribosenucleic acid, 124, 397

development, apomictic, 136

embryonic, 283

embryo-sac, 135

sexual, 135

vestigial tail, 283

diallel analysis, 1, 31, 420

cross, 413

modified, 32

differentiation, I14, 123

dinosaurs, 431

Diptera, 437

distyly, $219 \mathrm{sqq}$.

Dobzhansky, T., 277

dominance, $276,324,421,422$

analysis of, 1,387

in tetraploids, 219,223
Dowdeswell, W. H., 432

Dowrick, V. P. J., 2 19-236

dog, $44^{\circ}$

drift, genetic, 242,432

Drosophila, 1 24, 234, 267 sqq., 281 , 441

body size, 125

egg production, 125

melanogaster, 1 24, 243, 282, 422

subobscura, 282

duodenal ulcer, 126

dyad, $136,198,377$

ecology, 1 20, $43^{1}$

egg production, Drosophila, 125

poultry, 243

electrophoresis, 427

embryo-sac, I 35, I 42 sqq., 345

endomitosis, 155, 373

endosperm, 130 sqq., 140,367

hybrid, $195 s q q$.

environmental effect, I sqq., 334

epistasis, 35, 63, 224, 277

Ericales, $44^{\circ}$

Erigeron, 153

ethnology, $16 \mathrm{r}$

euchromatin, $196 s q q$.

euchromatic region, 85,368

Evans, D. A., 126

evolution, I I 9, 1 35, 254, 342 chromosome number, 284 mechanism of, 432

rate of, 121

\section{Fagopyrum, 231}

Falconer, D. S., 283

fall ratio, 24, 25

fertility, $132,221,233,284,431$

Acacia, 57 sqq.

human, 278

Trillium, 199, 202

fertilisation, genetic control of, 157 preferential, 254, 345 sqq., 353 sqq.

Festuca, 127, 199, 201,363

Feulgen technique, 368

Fincham, J. R. S., 279

Finney, J., 275

Fischberg, M., 284

Fisher, Sir Ronald, 35, 276, 432

fitness, 125

flagellates, 433

flowering time, Nicotiana, I sqq., 329 sqq.

Ford, C. E., 284, 440

Fritillaria, 399

fungus, 254

homothallic, 280

gametophyte, $377,4^{00}$

geitonogamy, 259

gene, action, 434

albino, Prunus, 242

complex, 227, 234

deleterious, 238

diffusion, $43^{2}$

duplicate, 4,27 sqq. 
gene, frequency, 237 sqq., $285,323,328$ lethal, 237, 263 sqq.

maps, 285

neutral, 238, 241

structure, 434

generation matrix, 33, 264 sqq.

genetic, assimilation, $28 \mathrm{I}$

background, 285

correlation, Drosophila, $28 \mathrm{I}$

drift, 96, 121, 242, 432

erosion, 237

isolation, 260

material, organisation of, 285

parameters, 31 sqq.

resolving power, 124

genoid, 435

genome, Trillium, 85

genotype-environment interaction, I, 22 sqq., 316

germination rate, 57 sqq., $\mathrm{I} 27$

Glovers, S. W., 28o

Godetia, 195, 201

Goldschmidt, R. B., 433-437

Goodspeed, T. H., I 19

Gossypium, 234

graft, cross, 280

Grassé, P.-P., 44I

Griffing, B., $3 \mathrm{I}-50$

Gruneberg, H., 283

Hackett, W. E. R., 69-84

hæemoglobins, 425 sqq.

hæmolysis, 73

Haga, T., 85-98

Hair, J. B., 129

Haldane, J. B. S., 123

Hammerton, J. L., 284, 440

Hardy-Wienberg law, 276

Hayman, B. I., 27 I

height, Nicotiana, I sqq., 329 sqq.

heritability, 282 sqq.

significance tests, I 24

hermaphrodite, 126

heterochromatic segments, 85

heterochromatin, $196,354,367$

in endosperm, 396

metamorphosis of, 373

heterokaryon, 244

heterosis, 5 sqq., 28, 341 $4^{\text {I I }}$

heterostyly, 219 sqq.

heterothallism, relative, 254

heterozygosis, 238 sqq., 263,282

heterozygote, duplex, 208 sqq., 224

interchange, 99

nulliplex, etc., 213

simplex, 208 sqq., 224

superiority, 237 sqq.

triplex, 208 sqq.

Hieracium, 153

Holden, J. W. H., 109

homeostasis, 238, 244, 283

homostyly, Primula, I 25, 219 sqq., 231

homozygosis, 269, $27 \mathrm{i}$

host effect, $28 \mathrm{I}$
H-segment, 267 sqq.

Hyacinthus, 389, 402

hybrid, structural, I 49

hybridisation, Trillium, $8_{5}$

identical twins, Agropyron, 147

Ikin, E. W., 425

inbred, cotton, 314

rye, I 12

inbreeding, $156,263,271,282,323,409$

coefficient, 431

depression, 67, 125, 340

progress, 125

theory, 276

incompatability, 125, 219 sqq., 260, 284 bracken, 247 sqq.

Nicotiana, I 19

inheritance, cytoplasmic, 435

extranuclear, 280

interaction, allelic, 234

genic, 323 sqq.

non-allelic, I, 4 I 3

interkinesis, $3^{88}$

inversion, $23 \mathrm{I}$

irradiation, high frequency, 123

gamma, 127

ultra-violet, 279

X-ray, 1 23, 28 I , 439

isochromosome, 386

isolation, sexual, $237,28 \mathrm{I}$

Jinks, J. L., I-30

Kacser, H., 285

Kafer, E., 279

Kammerer, 433

karyogamy, 254

Kettlewell, H. B. D., 287, 439

Knight, G. R., 28I

Lamarck, 432

Lebistes, 126

lethals, autosomal, 123

balanced, 265,267

dominant, 237

induced, 123

Lederberg, 435

Lehmann, H., 425

Lepidoptera, 287 sqq., 437, 439

L'Héritier, Ph., 43 I, 435

Li, C. C., 275, 432

lily, 403

linkage, $8,19,227,234,328,431$

Acacia, 63

group, 283

and inbreeding, 125

litter-size, mice, 283

locusts, 396

locus, sex-linked, 276

Lolium, 127

Luzula, I 4

Lycopersicum, 229

Lymantria, 435

Lythrum, 229, 276 
maize, see Zea

Malecot, $43^{2}$

male-sterility, I 57

man, 234, 431, $44^{\circ}$

races, I $6 \mathrm{I}, 285,425$

Manning, H. L., 303-322

map distance, 1 24, 205, 229

Mather, K., 271,276

mating, assortative, 323, 43 I

half-sib, 282

sib, 264, 323

selfing, 5I, 247, 263, 323

parent-offspring, 268

random, $5^{1}$ sqq., 323 sqq.

McConnell, R. B., I 26

Mecostetus, 399

meiosis, 99

in binucleate cells, 109 sqq.

maize, 354

non-synchronised, I 09 sqq.

suppression of, 133, I 57

melanism, industrial, 287, 439

Melanopus, 398, 399

methionine synthesis, 280

Mitchell, H. K., 120

micronucleus, I 13

mitosis, sensitivity of, 439

modifier, $28 \mathrm{I}$

Moffett, A. A., 57-67

Mole, R. H., $44^{\circ}$

Mota, M., 109

Mourant, A. E., 425

mouse, 234, 284 autosomal lethals, I 23 primary sex ratio, 126

mutagen, $270,3^{8} 5$

mutation, 276,28 I

lethal, 263

rate, 183,237 sqq., 403

Salmonella, I 23

natural crossing, estimation of, 57 sqq. segregation and, 60

natural selection, 41 3, 422

estimation of, $287 s q q$.

Narcissus, I 99 sqq., 388

Neurospora, 244, 279

newt, 284

Nicotiana rustica, I, I I 9, 328, 422

non-allelic interaction, I sqq., 81 sqq.

non-disjunction, 99, 354, 376

mitotic, 345 sqq.

numerical, $205 \mathrm{sqq}$.

Nordback, K., 28I

nuclei, competition between, I 3

generative, II 4

migrant, I 2

micro-, $148 s q q$.

polar, 321,380

restitution, 138

vegetative, I I 4

nucleolus, I 12, 435

nucleotide, 436

pair, 124
Ochna, I 46

Oehlkers, Fr., $43^{2}$

Enothera, I 06, 234

olisterochromatin, 367

Orthoptera, 437

Osborne, R., 286

ovary transplantation, $28 \mathrm{I}$

overdominance, $\mathrm{I}, 6,28,328,340,414,42 \mathrm{I}$

Owen, A. R. G., 432

panmixis, 318

Pantelouris, E. M., 28I

paramixis, 156

Paris, 201, 383, 402

Parthenium, 142

parthenogenesis, I 32 sqq.

Pateman, J. A., 279

path coefficient, 276

penetrance, 28I

Penrose, L. S., 43 I

pentasomics, 2I 5,2 I 6

phage, 280, 435

phenocopy, 28I

Philip, U., 126

Pirie, N. W., 43 I

Pisum, 99

plasmagene, 435

plasticity, 277

plastid, 435

pleiotropy, 243 sqq.

Poa, I56, 301

pollen mother cells, I 09, I 29, I47, I 53

pollen tube growth, 220, 255

polygenic inheritance, 440

polymorphism, 12 I, 237, 277, 440

polynemy, 44I

polyploidy, Trillium, $8_{5}$ sqq.

Pontecorvo, G., 124

population genetics, 275

size, 431

position effect, 234

poultry, 286

predation, 287 sqq.

Primula, 125, 219 sqq.

progeny test, I 25

prototroph, 279

Protozoa, 435

Prunus, 237 sqq.

pseudogamy, I34, I 55

Pteris, 253

pollen grains, maize, 357 sqq.

Quadling, C., 123

rabbit, $44^{\circ}$

Race, R. R., I 77, 285

radiomimetic substance, 393

reciprocal difference, $5,221,233,360$

recombination, 284,413

fraction, 268

frequency, 24, 53

value, 64

reduction, double, 205 sqq.

relational balance, $66,340,342$ 
Rees, H., 409-424

Reeve, E. C. R., 125, 282

Renner effect, I 36

reproduction, sub-sexual, I 29, I 57

Reusch, J. D. H., 127

Revell, S. H., I 23

Rhoeo, ro7

Rhyacia, 121

Robertson, A., 281, 282

Robertson, F. W., 125

Roper, J. A., I 24, 279

Rutishauser, A., I 95-204, 367-407

rye, $99 s q q ., 409 s q q$.

Salmonella, 1 23, 280

Sanger, R., 285

scaling tests, 2 sqq.

Scilla, I I 4, 389

scoring methods, 293 sqq.

seasonal effects, 20

Secale, 201,363

segregation, chromatid, 276

modes of, 205

polarised, $346 s q q$.

random, $346 s q q$.

tetraploid, 208

tetrasomic, 2 I 7

trisomic, 217

self-fertility, Primula, 223, 234

self-incompatability, 257 sqq.

selection, 66,12 I $, 254,28$ I

against homozygotes, $27 \mathrm{I}$

during inbreeding, 125

genetic response, 237

index, cotton, $303 s q q$.

industrial melanism, $287 s q q$.

intensity, 125

natural, 96, 413

stabilising, 244, 303

selective, advantage, 353,363

value, 277

serum, 427

groups, 73

sex-linkage, 276

sex ratio, primary, 126

sexual isolation, 237, 28 I

Sharpe, H. S., 280

sheep, $44^{\circ}$

Sheppard, P. M., I 26

sickle-cell, 425

Slizynski, B. M., 283

Smith-White, S., $44^{\circ}$

Smith, P. Maynard, 282

somatic aberrations, 354

Sorghum, 201

speciation, I i $9,121,342$

Trillium, 85,94

spindle, 435

pole, 345,346

Spurway, H., 126

stability, 28, 285, 414 sqq.

Stenobothrus, 399

sterility, 99

male-, 132, 157 sternopleural bristles, 283

Stocker, B. A. D., 123

surface specificity, 285

survival rates, $27 \mathrm{I}$

Tantawy, A. O., 282

tetrad analysis, 37 I

tetraploid ratios, 229

Thera, 12 I

Thoday, J. M., 283

Thompson, J. B., 99, 409

Tradescantia, 401, 402

transduction, 280

translocation, $283,284,386$

transplantation, tumour, $44^{\circ}$

Trillium, 85, I 95 sqq., 367 sqq.

Trimerotropis, 200

trisomics, 2 I 5

Triticum, 398

tumour, 440

twins, Agropyron, 147

urodele, 44 I

variation, $66,237,276$

additive genetic, $1,35,124,125$

continuous, 323

during inbreeding, $14,303,314$

environmental, 125, 323

estimation of components, 18

$F_{1}, 4^{1} 4$

free, 339

fixable, 323

genetic, in inbreds, 314

non-additive genetic, I, 35, 125

persistency, 318

potential, 339 sqq.

Venkateswarlu, J., 2 I 7

viability, 64, 216,282

relative, 233

Vicia, 123

viruses, 435

Waddington, C. H., $28 \mathrm{I}$

Wagner, R. P., I 20

Wallace, M. E., I 27

Watkin, I. M., I6I

Wilkie, D., 247

Wilkinson, G. N., 2 I 4

Williams, W., 237

Woolf, B., 124

Wright, Sewall, 277, 43 I

$\overline{\mathrm{W}}$-surface, 277

X-rays, 282

effect on chromatids, 123

mutation, 28I

recovery from, 28 I

Zea mays, 99, 195, 201, 205 sqq., 234, 345, 